\title{
BMJ Open Effect of wheelchair-modified rowing exercise on cardiometabolic risk factors in spinal cord injured wheelchair users: protocol for a randomised controlled trial
}

\author{
Rasmus Kopp Hansen (D) , ,2 Afshin Samani, ${ }^{1}$ Uffe Laessoe, ${ }^{2,3}$ Aase Handberg, ${ }^{4,5}$ \\ Ryan Godsk Larsen ${ }^{1}$
}

To cite: Hansen RK, Samani A, Laessoe U, et al. Effect of wheelchair-modified rowing exercise on cardiometabolic risk factors in spinal cord injured wheelchair users: protocol for a randomised controlled trial. BMJ Open 2020;10:e040727. doi:10.1136/ bmjopen-2020-040727

- Prepublication history and additional material for this paper are available online. To view these files, please visit the journal online (http://dx.doi org/10.1136/bmjopen-2020040727).

Received 20 May 2020 Revised 09 September 2020 Accepted 15 September 2020

Check for updates

(C) Author(s) (or their employer(s)) 2020. Re-use permitted under CC BY-NC. No commercial re-use. See rights and permissions. Published by BMJ.

For numbered affiliations see end of article.

Correspondence to Mr Rasmus Kopp Hansen; rkopp@hst.aau.dk

\section{ABSTRACT}

Introduction Cardiovascular and metabolic diseases are a growing concern for individuals with spinal cord injury (SCl). Physical inactivity contributes to cardiometabolic morbidity and mortality in the SCI population. However, previous studies have shown mixed results regarding the effects of exercise on cardiometabolic risk factors in individuals with $\mathrm{SCl}$. This discrepancy could be influenced by insufficient exercise stimuli. Recent guidelines recommend 30 min of moderate-to-vigorous intensity aerobic exercise, three times per week, for improvement in cardiometabolic health in individuals with $\mathrm{SCl}$. However, to date, no studies have implemented an exercise intervention matching the new recommendations to examine the effects on cardiometabolic risk factors. Therefore, the primary objective of this study is to determine the effects of 12 weeks of wheelchair usermodified upper-body rowing exercise on both traditional (constituents of the metabolic syndrome) and novel (eg, vascular structure and function) cardiometabolic risk factors in manual wheelchair users with SCl.

Methods and analysis A randomised controlled trial will compare 12 weeks of upper-body rowing exercise, 30 min three times per week, with a control group continuing their normal lifestyle. Outcome measurements will be performed immediately before (baseline), after 6 weeks (halfway), 12 weeks of training (post) and 6 months after the termination of the intervention period (follow-up). Outcomes will include inflammatory (eg, C reactive protein) and metabolic biomarkers determined from venous blood (with serum fasting insulin as primary outcome), body composition, arterial blood pressure, cardiorespiratory fitness level, brachial artery vascular structure and function and autonomic nervous system function. Ethics and dissemination This trial is reported to the Danish Data Protection Agency (J.nr. 2019-899/10-0406) and approved by the Committees on Health Research Ethics in The North Denmark Region on 12 December 2019 (J.nr. N-20190053). The principal investigator will collect written informed consent from all participants prior to inclusion. Irrespective of study outcomes, the results will be submitted to peer-reviewed scientific journals for publication.

\section{Strengths and limitations of this study}

- The frequency, duration and intensity of the exercise intervention follows recently published exercise recommendations for individuals with spinal cord injury.

- This study uses a randomised controlled design to examine the effects of a novel exercise modality on both traditional and novel cardiometabolic risk factors.

- The exercise modality (upper-body rowing) includes not only an aerobic component, but also an element of resistance training for the posterior shoulder region, potentially ameliorating shoulder pain.

- Lack of control of food intake is a study limitation as altered energy intake could influence the interpretation of the effects of exercise on body mass and body composition.

Trial registration number NCT04390087.

\section{INTRODUCTION}

Individuals with spinal cord injury (SCI) are placed at the lowest end of the fitness continuum. ${ }^{1}$ Thus cardiovascular disease and metabolic dysfunction are a growing concern in this population. ${ }^{23}$ In recent years, cardiovascular disease has emerged as the leading cause of mortality in individuals with chronic SCI. ${ }^{4}$ Factors contributing to high cardiometabolic morbidity and mortality in individuals with SCI are a sedentary lifestyle and low physical activity level, ${ }^{5}$ and the ensuing negative influence on body composition, reflected by lower fat-free mass and a larger amount of adipose tissue. ${ }^{6}$

The clinical manifestations of SCI rely on the level of neurological injury and the completeness of injury. ${ }^{7}$ SCI can result in complete or partial loss of sensorimotor function below the level of injury. In general, a 
cervical injury leads to impairment of all four extremities (ie, both arms and legs) as well as the trunk and pelvic organs (tetraplegia), whereas an injury to the thoracic, lumbar or sacral spine preserves the functioning of the arms (paraplegia). ${ }^{8}$ In addition to the impairment of sensorimotor function, SCI can disrupt sympathetic nervous system function as preganglionic sympathetic neurons are located between the T1 and L2 spinal segment. ${ }^{7}$ Due to an increased amount of paralysed muscle mass and larger disruption of the autonomic nervous system, lower physical capacity ${ }^{9}$ and heightened cardiovascular risk ${ }^{10}$ are generally observed among individuals with a high (tetraplegic) and complete SCI. The loss of sensorimotor function in the lower extremities forces many individuals with SCI to rely on a wheelchair for mobility. For those wheelchair users with sufficient strength and movement control in the arms, a manual wheelchair (ie, a wheelchair that can be propelled by the user) is typical used for mobility. ${ }^{11}$

Besides the obvious limitation to physical activities requiring ambulation, explanations for the adoption of a sedentary lifestyle are multifactorial, but studies have identified intrapersonal and socioenvironmental physical activity barriers among manual wheelchair users with SCI. ${ }^{12-14}$ Some barriers such as the intrapersonal barriers of lack of time ${ }^{15}$ or energy ${ }^{16}$ identified by individuals with mobility disabilities are consistent with those reported in the non-disabled population. Other barriers are specific to the mobility of individuals with a disability, such as organisational or structural barriers (eg, lack of accessible fitness centres ${ }^{12}$ and adaptive exercise equipment ${ }^{13}$ and community built environment barriers. ${ }^{17}$ Consequently, new approaches to support the initiation and perseverance of physical activity in this population are required.

The majority of previous exercise intervention studies have used isolated aerobic exercise, often in the form of arm-cranking exercise. ${ }^{18}$ These studies have demonstrated improvements in traditional risk factors for cardiometabolic diseases, such as high-density cholesterol (HDL-C) ${ }^{19}$ fasting insulin ${ }^{20}$ and indices of insulin resistance, ${ }^{21}$ whereas the effects on arterial blood pressure (BP), ${ }^{22}$ blood lipids (eg, low-density cholesterol (LDL-C) and triacylglycerol) are inconclusive. ${ }^{20} 2223$ Moreover, although evidence from cross-sectional studies suggests an association between increased participation in moderateto-vigorous intensity leisure-time physical activity and a reduction in visceral adipose tissue, ${ }^{24}$ there is insufficient evidence for the effects of upper-body aerobic exercise on changes in body mass and body composition, such as a reduction in total and visceral adipose tissue..$^{182526}$ The explanation for the lack of an exercise effect on some of these above-mentioned risk factors is not clear, but could be related to insufficient volume ${ }^{2022}$ or intensity ${ }^{25}$ of exercise, performed with a limited amount of skeletal muscle mass.

Notably, in able-bodied individuals, the exerciseinduced risk reduction in cardiovascular diseases cannot be fully explained by traditional risk factors (constituents of the metabolic syndrome), that is, there is a risk factor gap. ${ }^{27}$ As a consequence, studies have started to focus on the effects of exercise on changes in the vascular wall. ${ }^{27}$ It is known that dysfunction of the vascular endothelium occurs at the very early phases of atherosclerosis. ${ }^{28}$ For instance, flow-mediated dilation (FMD), a non-invasive measure of nitric oxide dependent endothelial function, ${ }^{29}$ is a strong predictor of future cardiovascular events. ${ }^{30}$ In addition, carotid intima-media thickness (IMT), a measure of vessel wall thickness ${ }^{31}$ has shown to be associated with future vascular events, such as the occurrence of stroke and myocardial infarction. ${ }^{31}$ Accumulating evidence in able-bodied individuals demonstrates beneficial effects of exercise on structural and functional adaptations of the vasculature. ${ }^{32}$ However, little is known about the effects of exercise on the vasculature in individuals with SCI.

Reductions in the femoral artery (lower body) diameter occur rapidly in response to extreme inactivity, as observed within 3 weeks of acquiring an SCI. ${ }^{33}$ De Groot $e t$ $a l^{34}$ found similar FMD in the brachial artery (BA) among untrained SCI when compared with able-bodied individuals, however, when FMD was normalised to the shear stress stimulus, the dilation response was reduced in the SCI group, indicating some degree of endothelial dysfunction in this population. ${ }^{34}$ Other observational studies have demonstrated both larger conduit artery diameter and blood flow in the subclavian artery, ${ }^{35}$ and larger brachial diameter ${ }^{36}$ in wheelchair athletes compared with non-athlete able-bodied controls. Together these findings indicate that remodelling of the vasculature also occurs in response to regular exercise in wheelchair users with SCI. However, the causal link between adaptations of the upper body arteries and repetitive exercise stimulus in SCI is not fully established. Therefore, the relationship between exercise and vascular remodelling in manual wheelchair users needs to be determined through controlled exercise studies. A recent experimental study consisting of supervised aerobic exercise (20 min, two times per week), adhering to the earlier (2011) exercise guidelines for adults with SCI, ${ }^{37}$ was successful in improving measures of cardiorespiratory fitness and muscle strength. ${ }^{38}$ Yet, Totosy de Zepetnek et $a l^{22}$ demonstrated that this exercise paradigm (20 min, two times per week) did not improve markers of cardiovascular disease risk, including carotid IMT and brachial FMD. Recent (2018) guidelines recommend at least $30 \mathrm{~min}$ of moderate-to-vigorous intensity aerobic exercise, three times per week in order to improve cardiometabolic health in individuals with SCI, ${ }^{39}$ suggesting that insufficient exercise stimuli may explain the lack of vascular adaptations in the study by Totosy de Zepetnek et $a l .^{22}$ Notably, to date, no studies have examined the effects of the updated exercise guidelines (30 min of moderate-to-vigorous intensity aerobic exercise, three times per week) on vascular adaptations in manual wheelchair users with SCI.

While isolated aerobic exercise, via arm-cranking or wheelchair ergometry, evokes positive effects on 
cardiorespiratory fitness, these modalities/types of exercises fail to address the importance of strengthening the posterior shoulder musculature of the upper-extremity for individuals with SCI relying on a manual wheelchair for mobility. ${ }^{40}$ In addition, these modalities use repetitive contractions of the shoulder musculature engaged in daily wheelchair propulsion activities, thereby increasing the risk of developing shoulder pain. ${ }^{41-43}$ Studies have reported a high prevalence of shoulder pain in long-term wheelchair users, ${ }^{45}$ which prevents these individuals from engaging in physical activity. ${ }^{46}$ As wheelchair users rely on their upper-extremities for most daily activities, upper-extremity pain must be prevented or limited to preserve function, independence and quality of life (QOL) ${ }^{21}{ }^{47}$ Indeed, health-related QOL is lower in adults with SCI compared with the able-bodied population. ${ }^{48}$ The development of shoulder pain has been suggested to occur due to chronic overuse, shoulder strength imbalances between anterior and posterior musculature, postural changes and impingement syndrome. ${ }^{50-52}$ Considering the general need for strong upper extremities to bear weight during different transfer tasks,${ }^{53}$ to propel the wheelchair ${ }^{54}$ and to reach overhead levels, ${ }^{55}$ the inclusion of resistance training in exercise training paradigms seems prudent. One modality that combines aerobic and resistance components is rowing. ${ }^{56}$ Specifically, it has been shown that wheelchair user-modified upper-body rowing ergometry challenges the cardiovascular system comparable to arm-cranking. ${ }^{41}$ Additionally, upper-body rowing mirrors the muscle activation observed during traditional resistance training for the scapular retractors. ${ }^{57}$ Earlier research has demonstrated beneficial effects of resistance training of the posterior shoulder and scapular retractor musculature on shoulder pain. ${ }^{43} 4755$ Thus, the use of upper-body rowing ergometry may evoke positive effects on both cardiometabolic health and shoulder pain in manual wheelchair users, the latter through alterations in posterior vs anterior upperbody muscle strength balance. However, to date, no studies have implemented upper-body rowing ergometry as exercise modality in this population.

\section{Objectives}

The primary objective of this study is to determine the effects of 12 weeks of wheelchair user-modified upperbody rowing on both traditional (insulin resistance, obesity, dyslipidaemia (including low HDL-C and elevated triglycerides (TG)), and $\mathrm{BP}$ ) and novel (inflammatory status, autonomic nervous system function, vascular structure and function and cardiorespiratory fitness level) cardiometabolic risk factors in manual wheelchair users with SCI. As secondary objectives, we will investigate the effects of the exercise intervention on leisure-time physical activity, shoulder pain, indices of QOL and feasibility of the intervention.

\section{METHODS AND ANALYSIS \\ Study design}

A randomised controlled trial designed to determine the effects of 12 weeks of exercise training on cardiometabolic risk, indices of QOL and shoulder pain, will be conducted in accordance with the Standard Protocol Items: Recommendations for Interventional Trials statement. ${ }^{58}$ The trial is registered as a controlled trial on 15 May 2020 with first enrolment beginning in November 2020. The study overview is presented in figure 1 . After giving written informed consent to participate in the study (see online supplemental appendix for details about the model consent form), participants will undergo baseline testing, after which they will be randomly assigned to either a control group or an exercise group (allocation ratio, 1:1), stratified for age, self-reported leisuretime physical activity level and SCI level. Randomisation

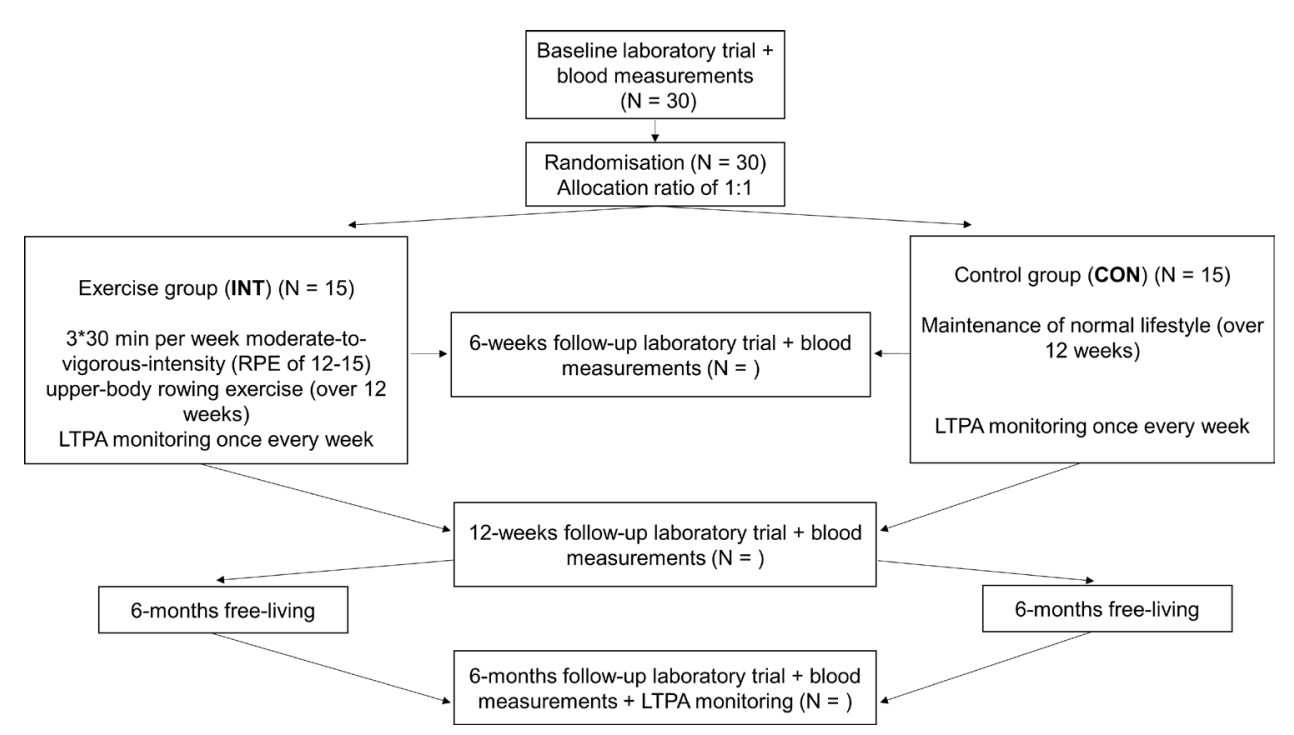

Figure 1 Study overview. CON, control; INT, intervention; LTPA, leisure-time physical activity; RPE, rating of perceived exertion. 
will be conducted using a computer-generated random number sequence (https://www.randomizer.org/). Outcome measurements will be performed immediately before (baseline), after 6 weeks (halfway), 12 weeks of training (post) and 6 months after the termination of the intervention period (follow-up). This approach allows for assessment of the short term effects of exercise training as well as any residual effects from the training intervention on cardiometabolic risk, shoulder pain, indices of QOL and self-reported leisure-time physical activity. Participants will be asked to maintain their normal dietary habits throughout the study period.

\section{Participants}

\section{Inclusion criteria}

Men and women; aged 18-65 years; chronic SCI ( $\geq 1$ year since injury); individuals with sufficient sparing of arm flexor function to participate in upper-body rowing (ie, as a minimum excluding individuals with complete SCI at or above C5); using a manual wheelchair as a primary tool for mobility. Volunteers will be asked to self-report SCI level and completeness and to provide a copy of the part of their medical records that confirm injury level and American Spinal Injury Association impairment scale classification before any measurements are commenced. In addition, volunteers will be asked to briefly perform the rowing exercise during the first laboratory visit to ensure that the individual is able to perform the exercise intervention. As part of the screening, volunteers will also be asked to indicate smoking habits, known medical issues, diseases or use of medication that could affect metabolism (eg, statins or metformin) or the cardiovascular system (eg, diuretics, beta-blockers or angiotensinconverting enzyme inhibitors).

\section{Exclusion criteria}

Individuals who regularly engage in $>90 \mathrm{~min} /$ week of moderate-to-vigorous intensity physical activity; have received a cortisone injection in the shoulder within the last 4 months; have had shoulder injury within the previous year; known medical issues (urinary tract infections, cardiovascular contraindications for exercise testing and pressure sores); diagnosed diabetes or any other disease that may limit the ability to perform exercise.

\section{Recruitment}

Starting from September 2020, participants will be recruited through notices at Aalborg University, websites seeking volunteers for research studies (eg, www.forsog. $\mathrm{dk}$ ), organisations related to disability and SCI, including the Spinal Cord Injured in Denmark organisation, physician clinics, wheelchair manufacturing company (Wolturnus $\mathrm{A} / \mathrm{S}$ ) and local community groups. Information on websites, social media, posters, and flyers will be used to reach potential participants. This kind of participant recruitment can be considered passive since the participants have to choose to react to the study information. ${ }^{59}$ This approach increases the risk of over-representing individuals who are interested in the research area ${ }^{59}$ Considering this risk of selection bias, physician clinic visits from the principal investigator (ie, active recruitment) will be used as an additional strategy to reach potential participants. For this study, 30 participants will be recruited. The sample size is based on the effect size (Cohen d: -0.69) for changes in fasting insulin after 6 weeks of arm-cycling exercise. ${ }^{20}$ With a power of 0.9 , and an alpha level of $0.05,20$ participants in total are required to detect a significant change. To account for drop-outs during the 12-week intervention, as well as potential drop-outs in the control group, 30 participants will be included in the project, with $\mathrm{n}=15$ allocated to the control and exercise group, respectively.

\section{Intervention}

\section{Exercise group}

Volume, intensity and frequency of the intervention are based on recent exercise recommendations for individuals with SCI. ${ }^{39}$ Consequently, the training will be performed for $30 \mathrm{~min}$, three times per week with moderate-to-vigorous intensity, with at least one rest day between sessions. A low compliance rate is a general issue in SCI exercise studies ${ }^{60}$ Compared with continuous exercise, interval-based exercise has been reported to elicit higher enjoyment in individuals with SCI, ${ }^{61}$ which may increase exercise compliance. Considering this difference between continuous and interval-based exercise, the target duration of $30 \mathrm{~min}$ will be reached through the accumulation of 5-minute bouts (up to six bouts), with 1-2 min of rest between each bout. It is possible that some participants will not be able to exercise for $30 \mathrm{~min}$ with the intended intensity. Accordingly, exercise duration will be tailored to each participant's physical capacity ${ }^{62}$ by gradually increasing the duration (ie, the number of 5-minute bouts) over the weeks towards the targeted 30 min (ie, six * 5-minute bouts). Exercise intensity will be prescribed based on the rating of perceived exertion (RPE) corresponding to 12-15 on the 'Borg 6-20 RPE Scale. ${ }^{63} 64$ The validity of using RPE to control moderate and vigorous exercise intensity has been demonstrated in individuals with SCI. ${ }^{65}$ During the first visit, participants will be familiarised with the RPE scale ${ }^{66}$ and receive detailed instructions on the use of RPE and how to rate the overall exertion based on an integration of central and peripheral sensations of effort. ${ }^{65}$ Some controversy exists about the validity of using overall RPE to monitor exercise intensity, ${ }^{66} 67$ as the relationships between overall RPE and objective physiological markers such as oxygen consumption $\left(\mathrm{VO}_{2}\right)$, heart rate $(\mathrm{HR})$ and ventilation in individuals with SCI have been questioned. ${ }^{67}$ This has led some researchers to use a differentiated RPE scale that distinguishes between central (cardiorespiratory sensations) and peripheral (peripheral working limbs) sensations. ${ }^{68}$ However, the current evidence does not indicate greater validity for differentiated RPE compared with overall RPE ${ }^{66}$ and therefore overall RPE will be used to guide exercise intensity. 
Each training session will consist of aerobic exercise performed on a wheelchair-modified upper-body rowing ergometer (Concept 2, Morrisville, Vermont, USA). Due to the pulling motion of the upper-body during rowing, this modality also includes a component of resistance exercise. ${ }^{56}$ The modification of the ergometer is made by separating it into two parts using an Adapt2row (http:// www.adapt2row.com/), allowing the participants to sit in their wheelchair while performing upper-body rowing. Wedges will be positioned under the rear wheels to keep the wheelchair in place. Further, in case of SCI related insufficient innervation of torso musculature, straps will be wrapped around the back of the wheelchair and around the trunk of the participant, thereby securing the participant to the wheelchair. A pilot study ${ }^{41}$ has provided promising results regarding the feasibility of using adaptive upper-body rowing exercise in the SCI population. Participants will be asked to empty their bladder before each training session. Average power output (W) will be recorded during each exercise session to monitor the training load and quantify progress in work capacity.

All exercise sessions will be supervised by the principal investigator, who is an exercise physiologist or by sport science students to ensure proper assistance and guidance of the participants. Everyone involved in supervision will be thoroughly instructed on how to supervise the correct exercise technique, and they will receive general knowledge about SCI and wheelchair use. To allow for some flexibility regarding scheduling conflicts, participants will have the opportunity to exercise $2-4$ days per week, with a target of 3 days per week. That is, in case of missing a training session, an additional session can be included the following week. However, to secure adequate recovery, no more than four training days per week will be allowed. The intervention will be terminated after 12 weeks, irrespective of any missing training sessions. An adherence rate threshold of $75 \%$ will be used ( $\geq 27$ exercise sessions out of the maximum 36) ${ }^{69}$ However, there are no previous reports of adherence rates for this type of exercise intervention (12 weeks of wheelchair-modified rowing) in individuals with SCI. Therefore, if some participants show low $(<75 \%)$ adherence rates, we intend to do a sensitivity analyses in order to determine how sensitive the exercise responses are to reaching $(\geq 75 \%)$ or not reaching $(<75 \%)$ the a priori set adherence rate. If the adherence rate influences the exercise response, we will consider including the adherence rate as a covariate in the statistical analyses.

\section{Control group}

The participants allocated to the control group will be asked to maintain their normal lifestyle throughout the intervention period. When the 6 months follow-up testing has been terminated, the control group will receive information about the exercise guidelines and the benefits of physical activity. Additionally, if requested, the principal investigator will organise contact to facilities where participants in the control group can perform adapted rowing.

\section{Main trial day protocol}

The experimental procedures will be similar at baseline, halfway, postintervention and at follow-up 6 months later. To account for within-day variation, the procedures will be performed at the same time of day for each participant. Participants will arrive at the exercise laboratory at Aalborg University for testing. Participants will be asked to refrain from any strenuous exercise ( $>24$ hours), caffeine, alcohol, polyphenols, vitamin $\mathrm{C}$ and supplements known to affect the cardiovascular system ( $>12$ hours) and attend in a fasted ( $>6$ hours) state. ${ }^{70}$ Any smokers must abstain from smoking for $>6$ hours prior to each visit. ${ }^{70}$ If participants are taking medication, a wash-out period of at least four to five times the half-life of the drug (typically 24-48 hours) will be used. If a drug cannot be withdrawn due to health issues, testing will be performed after a consistent time period after intake, as recommended for studies examining vascular function. ${ }^{70}$ On trial days, participants will receive a standardised snack (energy bar) after completion of the FMD measurement. Participants will be asked to empty their bladder before any testing is commenced, and are allowed to drink water ad libitum during the test days.

\section{Blood collection procedure}

On a separate day, after an overnight fast (ie, $\geq 10$ hours of fasting), participants will have approximately $20 \mathrm{~mL}$ blood drawn from a peripheral vein. Blood samples will be aliquoted and stored at $-80^{\circ} \mathrm{C}$ at the Department of Clinical Biochemistry, Aalborg University Hospital, until analyses. Blood samples will be analysed for indicators of cardiometabolic health (markers of glucose homeostasis, dyslipidaemia, non-alcoholic fatty liver disease (NAFLD) and prothrombotic risk) as well as inflammatory markers (proinflammatory and anti-inflammatory markers). Blood sampling will be conducted within a week prior to commencing the intervention (baseline), between 36 and 60 hours after the last exercise session in week 6 (halfway) and week 12 (post) to minimise any effects from the last exercise session, and 6 months after the termination of the intervention (6 months follow-up).

\section{Outcome measures}

\section{Primary outcome}

The primary outcome is fasting insulin, since it is one of the hallmarks of the metabolic syndrome, ${ }^{72} 73$ and has shown to be modifiable with exercise training in individuals with SCI. ${ }^{20}$

\section{Secondary key outcomes \\ Arterial $B P$ and resting $H R$}

After resting for $10 \mathrm{~min}$, participants will have their resting systolic and diastolic $\mathrm{BP}$ and $\mathrm{HR}$ measured with an automated BP monitoring device (OMRON M3, OMRON Healthcare, Hoofddorp, Netherlands). Measurements will be performed two times, with participants in a sitting position. If these measurements deviate $>5 \%$, a third 
measurement will be performed. The lowest values will be used for further analyses.

\section{Body composition}

Participants will have their body mass measured while sitting in their wheelchair (wearing light clothing) using a platform wheelchair scale (Detecto 6550 wheelchair scale, Webb City, Missouri, USA). Body mass will be derived by subtracting the weight of the wheelchair from the total mass and rounded to the nearest $0.1 \mathrm{~kg}{ }^{74}$ Supine height $(\mathrm{cm})$ will be measured in a supine position using non-elastic tape. For participants with contractures precluding stretching of the legs, the length will be measured in segments from heel to top of the skull. ${ }^{75}$ Body mass index will be calculated as weight $(\mathrm{kg})$ divided by height squared $\left(\mathrm{m}^{2}\right)$. Waist circumference and waistto-hip circumference ratio will be used as a surrogate for visceral adiposity. ${ }^{74-76}$ Participants will have their waist and hip circumference measured in supine position following a deep expiration. Waist and hip circumference will be measured immediately below the lowest rib $^{75}$ and widest part of the trochanters, ${ }^{76}$ respectively. For all circumference measurements, the tape will be placed directly on the skin with the participants arms by the side. ${ }^{75}$ Anthropometric measures will be taken in duplicate (height, waist and hip circumference), rounded to the nearest 0.1 $\mathrm{cm}$ and reported as the mean. If the difference between the first and second measure is $>0.1 \mathrm{~cm}$, a third measure will be obtained. ${ }^{75}$

\section{Autonomic nervous system function}

Increasing evidence suggests that autonomic dysfunction accentuates the risk for adverse cardiovascular events. ${ }^{77}$ Individuals with SCI are prone to autonomic disturbances as a consequence of disruption to the spinal cord, ${ }^{7}$ placing them at increased risk for autonomic-related cardiovascular diseases. It is generally accepted that the heart in individuals with SCI below T6 is innervated by both sympathetic and parasympathetic neurons. In contrast, due to disruption of sympathetic outflow, parasympathetic innervation dominates in individuals with complete cervical and upper thoracic injuries, thereby resulting in bradycardia, reduced cardiac output and arterial BP. ${ }^{7}$ Recently, it has been shown, however, that sympathetic control can be partially preserved in athletes with a cervical motor complete injury, with the degree of preservation being an important determinant of exercise performance. ${ }^{78}$ Yet, whether exercise training can alter autonomic nervous system function (eg, balance between sympathetic and parasympathetic activity) in individuals with SCI is uncertain. Thus, as assessment of autonomic nervous system function, HR variability $(\mathrm{HRV})^{77}{ }^{79}$ and arterial BP changes in response to an orthostatic challenge (sit-up tilt test ${ }^{78}$ will be measured as exploratory aims. For HRV measurements, participants will be equipped with a four-lead surface electrodes on their chest and have their ECG recorded (LabScribe V.4, iWorx, Dover, Hampshire, USA) during 5 min of quiet rest. ECG data will be exported to dedicated software (Kubios HRV Standard V.3.2.0; Kuopio, Finland) for analyses of frequency-domain parameters (low-frequency power, high-frequency power and total power) and timedomain parameters (HR and the root mean square of successive RR interval differences) in accordance with guidelines from The European Society of Cardiology and Heart Rhythm Society. ${ }^{80}$ For the sit-up tilt test, participants will be equipped with a finger plethysmograph (Finometer, Finapres Medical Systems BV, Enschede, the Netherlands) for continuous and non-invasive measurement of arterial BP and HR during a 10 min orthostatic challenge (sit-up test). Briefly, after 10-15 min of supine rest with baseline recordings, participants will be moved (ie, without assistance from the participant) to an upright seating position with their legs hanging free of the bed at an angle of $90^{\circ} .^{78}$ Changes in systolic BP and diastolic BP will be calculated as the difference between mean seated and supine BPs. ${ }^{82}$ The presence of orthostatic hypotension will be defined as a $\geq 20 \mathrm{~mm} \mathrm{Hg}$ drop in systolic BP or $\mathrm{a} \geq 10 \mathrm{~mm} \mathrm{Hg}$ drop in diastolic BP when moving to an upright position. ${ }^{83}$

\section{Vascular structure and function}

After a rest period of 10-15 min in a quiet and darkened room, vascular structure and function will be evaluated non-invasively using ultrasonography (LOGIQ S8 XDclear, GE Healthcare) following recent guidelines on assessment of conduit ${ }^{70} 84$ and resistance ${ }^{71}$ vessel function. Conduit artery structure will be determined in the common carotid artery (CCA) and BA with B-mode echoes using a $10 \mathrm{MHz}$ multifrequency linear assay ultrasound probe. Measures of CCA and BA structure will be reported as IMT $(\mathrm{mm})$, lumen diameter $(\mathrm{mm})$ and wallto-lumen ratio. The B-mode image will be optimised by changing depth and resolution, and will be kept constant between study visits.

To assess BA and resistance vessel function, endothelialdependent FMD and reactive hyperemia will be determined by cuff occlusion followed by reperfusion. ${ }^{29}$ First, participants will have their baseline BA diameter measured for a period of $30 \mathrm{~s}$. Then, a cuff placed distally on the forearm (ie, distal to the ultrasound probe) will be inflated for $5 \mathrm{~min}$ up to a pressure that exceeds systolic BP by $>50 \mathrm{~mm} \mathrm{Hg}$. After $5 \mathrm{~min}$, the cuff will be deflated and postdeflation diameter and blood velocity will be continuously recorded for $3 \mathrm{~min} .{ }^{70}$ For determination of FMD, both absolute $(\mathrm{mm})$ and relative $(\%)$ change in diameter from baseline to postdeflation will be calculated. Given the importance of shear stress as the stimulus for the FMD response ${ }^{85}$ continuous and simultaneous measurement of pulse-wave velocity (Doppler) and diameter (B-mode) using duplex ultrasound will be performed. This allows for an estimation of the shear stress stimuli through the calculation of shear rate, which then can be used to normalise the FMD response. ${ }^{86}$ Because the error of the insonation estimation increases exponentially with angles $>60^{\circ},{ }^{87}$ an insonation angle of $\leq 60^{\circ}$ will be used, 
with the sample volume adjusted to cover the total width of the vessel.

Resistance vessel function will be determined by the magnitude of the reactive hyperemic response to cuffinduced ischaemia. Several measures obtained from the reactive hyperemic response will be reported, including absolute blood flow (calculated based on the diameter derived cross-sectional area and blood velocity) and velocity, peak change in blood flow and blood flow area under the curve across the postdeflation time period.

Although related, the measure of reactive hyperemia mirrors the magnitude of downstream resistance artery dilation, whereas FMD represents conduit artery dilation. ${ }^{71}$

To ensure that the same part of the CCA and BA will be insonated across study visits, anatomical landmarks will be identified and recorded together with a photo of the insonation sites. All insonation settings (including sample volume, insonation angle and recording time) and occlusion procedure (including cuff-position, pressure and duration) will be held consistent within participants across study visits. For standardisation, the same investigator will perform all measurements and all analyses (blinded to the identity of the data).

\section{Metabolic and inflammatory profile}

The metabolic and inflammatory profile will be assessed via measures of glycaemic control (fasting glucose, longterm blood glucose (HbAlc, glycosylated haemoglobin), homeostatic model assessment of insulin resistance), lipid profile (HDL-C, LDL-C, total cholesterol and TG), proinflammatory (C reactive protein, interleukin (IL) 6 , tumour necrosis factor-alpha), anti-inflammatory (IL-10, IL1RA), prothrombotic (PAI-1), markers of NAFLD (hepatic enzymes (alanine-aminotransferase and aspartate-aminotransferase)), growth differentiation factor 15 and fibroblast growth factor 21.

\section{Cardiorespiratory fitness level}

Cardiorespiratory fitness level will be determined through an incremental arm-cranking test to exhaustion, with peak oxygen consumption $\left(\mathrm{VO}_{\text {2peak }}\right)$ and ventilatory thresholds (VTs) as the outcome measures. The placement of the ergometer (Monark 881E, Vansbro, Sverige) will be adjusted such that the participants shoulder joint is aligned with the crank axis with the elbows slightly bend. Participants will be equipped with an HR monitoring belt (Suunto Ambit3 Run; Suunto, Vantaa, Finland) and a face mask, connected to an online open-circuit metabolic cart. The cart (JAEGER, Vyntus CPX, Carefusion) will be calibrated to known volumes and gas concentrations according to manufactural guidelines. After a 1 min warm-up with zero resistance, the test begins with an individualised starting load $(5-90 \mathrm{~W})^{88}$ with increases in workload $\left(5\right.$ or $10 \mathrm{~W}$ ) every minute ${ }^{89}$ until volitional fatigue, defined as an inability to maintain cadence above 55 rounds per minute $(\mathrm{rpm}){ }^{88}$ The individual starting load and increment size will be chosen based on training history and anticipated physical capacity, with the aim of reaching exhaustion within 8-12 min at a cadence of $60-70$ rpm. ${ }^{89}{ }^{90}$ Breath-by-breath $\mathrm{VO}_{2}$ and carbon dioxide output $\left(\mathrm{VCO}_{2}\right)$, and $\mathrm{HR}$ will be measured continuously throughout the test. $\mathrm{VO}_{2 \text { peak }}$ will be reported in both absolute $\left(\mathrm{L} \mathrm{O}_{2} / \mathrm{min}\right)$ and relative $\left(\mathrm{mL} \mathrm{O}_{2} / \mathrm{kg} / \mathrm{min}\right)$ terms, and defined as the highest $30 \mathrm{~s}$ average during the test, with the corresponding HR reported as $\mathrm{HR}_{\text {peak }}$. The highest workload that is achieved for $\geq 30$ s will be reported as $\mathrm{PO}_{\text {peak }}$. Participants will be asked to indicate RPE (Borg 6-20 scale) during the last $15 \mathrm{~s}$ of each minute. On test cessation, participants will gradually cool-down for $5 \mathrm{~min}$, while their BP will be measured immediately before and after this recovery period.

As part of the cardiorespiratory fitness assessment, the first VT $\left(\mathrm{VT}_{1}\right)$ and the second VT $\left(\mathrm{VT}_{2}\right)$ will be determined during the incremental arm-cranking test. $\mathrm{V}_{1}$ and $\mathrm{V}_{2}$ represent distinct physiological events, but are both related to an increase in blood lactate and a subsequent increase in $\mathrm{VCO}_{2}$ in response to increased exercise intensity. ${ }^{91}$ Each VT will be defined according to the V-slope method or the ventilatory equivalent method, depending on which plot most clearly illustrates the particular VT. ${ }^{91}$ In addition, a plot showing the respiratory exchange ratio will be used as an extra reference in order to support the recognition of individual thresholds. ${ }^{91}$ Thresholds will be determined independently by two researchers, with the average value used for analysis. ${ }^{92}$ In case of deviation of $>5 \%$ for individual thresholds, the disparity will be discussed and a mutual agreement will be found. ${ }^{92}$ Although $1 \mathrm{~min}$ stages seems short for the attainment of steady state, the usage of $1 \mathrm{~min}$ stages have previously shown to be efficient for the detection of VTs on a group level, especially in paraplegics. ${ }^{91}$

\section{Other secondary outcomes}

\section{Shoulder pain}

The prevalence and severity of shoulder pain will be assessed using the Danish version ${ }^{93}$ of the Wheelchair Users Shoulder Pain Index (WUSPI)). ${ }^{94}$ WUSPI is a valid and reliable measure of shoulder pain in manual wheelchair users ${ }^{51}$ The questionnaire uses a series of visual analogue scales ranging from 'no pain' to 'worst pain ever experienced', and it is a self-reported measure of the prevalence and severity of shoulder pain during different activities, such as dressing, bathing, transfer, wheeling up and sleeping. ${ }^{94}$ Some participants may not perform all of the 15 activities. To account for that, a performance-corrected shoulder pain score (PC-WUSPI) will be calculated by dividing the raw total WUSPI score by the number of performed activities, multiplied by $15 .^{95}$

\section{Health-related quality of life (HRQOL)}

HRQOL will be monitored using the Danish translated version of the Short Form-36 (SF-36) ${ }^{96}$ which is a reliable and validated questionnaire to assess HRQOL within both a physical and mental health domain. ${ }^{97}$ Certain questions of the SF-36 will be adapted to wheelchair use. Specifically, 
the questions referring to 'walk' and 'stair-climbing' is substituted by the words 'climb' and 'go up', as previously recommended. ${ }^{98}$ Construct validity stays acceptable with this modification. ${ }^{98}$ Data will be scored using the RAND 36-item Health survey 1.0 method, ${ }^{99}$ in which original responses are transformed into a score from 0 to 100 , with 100 representing the best possible health. Individual item scores are then averaged within domains to create eight subscales, four representing physical QOL and four representing mental QOL. For reporting of HRQOL, a physical component summary score and a mental component summary score will be created based on the average of each component subscales. ${ }^{100}$

\section{Leisure-time physical activity}

To determine the effects of the exercise intervention on short-term (6 and 12 weeks follow-up) and long-term (6 months follow-up) habitual physical activity, leisuretime physical activity levels will be monitored using the Leisure Time Physical Activity Questionnaire for People with Spinal Cord Injury (LTPAQ-SCI). ${ }^{101}$ In brief, the LTPAQ-SCI is a valid and reliable self-reported measure of leisure-time physical activity that assesses minutes of mild, moderate and heavy intensity leisure-time physical activity performed over the previous 7 days. ${ }^{101}$ Participants in both the intervention and the control group will be asked to complete the LTPAQ-SCI once every week throughout the 12 weeks intervention period and again once during the week leading up to the 6 months follow-up. In addition, completion of the LTPAQ-SCI will work as a training diary for the intervention group, allowing us to monitor leisure-time physical activity performed beyond the amount related to the exercise intervention, which will strengthen our ability to interpret the results from the intervention.

\section{Feasibility and acceptability of the exercise intervention}

Satisfaction with the exercise intervention will be evaluated with the Feasibility and Acceptability Questionnaire. ${ }^{102}$ In brief, participants will be asked to rate how feasible and acceptable they consider the rowing exercise to be on a Likert scale ranging from 1 (strongly disagree) to 5 (strongly agree). The questionnaire consists of six questions addressing issues such as how fun the exercise was perceived; the difficulty level of the exercise and whether they received appropriate guidance in how to perform the exercise. A mean score of $\geq 3.0$ will be used as a criterion to indicate that the intervention is acceptable. ${ }^{102}$ Additionally, compliance with the exercise intervention will be recorded and presented as a compliance rate (\% participation). Compliance is defined as the number of exercise sessions completed out of the total number (36) of sessions.

\section{Statistics}

The between-group difference in changes of outcome variables will be examined from baseline to 6 weeks follow-up (secondary endpoint), from baseline to postintervention
(12 weeks follow-up; primary endpoint) and again from postintervention to follow-up 6 months later (secondary endpoint).

Primary and secondary endpoint data are ratio and interval data and will, therefore, be treated as continuous variables. Between-group comparisons on the primary and secondary endpoint will be dependent on data distribution. We anticipate data to be approximately normally distributed; however, before any analyses are commenced, distribution of the data will be assessed through visual inspection of $\mathrm{Q}-\mathrm{Q}$ plots and histograms, complemented by a test of deviation from normality (Shapiro-Wilk test). Assuming normality, descriptive data will be presented as mean $\pm \mathrm{SD}$, and a two-way analysis of variance (ANOVA) with repeated measurements will be used to evaluate any significant changes in outcomes between (control and exercise) and within (baseline, 6 weeks and 12 weeks follow-up) groups from baseline to immediately after the 12 weeks intervention period. The same procedure will be done for the 6 months follow-up (factor 1: group (control and exercise), factor 2: time (baseline, 12 weeks and 6 months follow-up). Interactions between the factors will be included in the model. In case of significant F-values, post hoc testing will be used for multiple comparisons. The effect size of change scores (Cohen's d) will be calculated in order to determine the magnitude of difference between groups. $\mathrm{P}$ values and $95 \%$ CIs will be reported to facilitate interpretation of the results. Statistical significance will be accepted at $\mathrm{p}<0.05$.

\section{Patient and public involvement}

Neither participants nor public were involved with the conception of the research question, study design or outcome measures, nor will they be involved with study conduction. The recruitment plan was partially planned based on feedback from the patient population. Each of the participants will receive a written summary of the study results after publication.

\section{Ethics and dissemination}

Considerations are made regarding the regulation of arterial BP in the participants. ${ }^{103104} \mathrm{BP}$ instability does occur in cervical injured individuals (tetraplegics), who may suffer from pronounced autonomic disturbances. ${ }^{103} 104$ During the $\mathrm{VO}_{2 \text { peak }}$ test and exercise sessions, special attention is devoted towards potential symptoms of autonomic dysreflexia $(\mathrm{AD})^{105}$ in individuals with $\mathrm{SCI} \geq \mathrm{T} 6$. Participants will be asked to empty their bladder before any testing or training is commenced. In case of $\mathrm{AD}$, the exercise is stopped and the pertinent actions are made. Occurrences of postexercise hypotension ${ }^{104}$ and orthostatic hypotension ${ }^{103}$ are rather uncommon in individuals with thoracic injury (paraplegics). According to the inclusion criteria of the study, only individuals with a sparing of arm flexor function (ie, excluding individuals with a complete cervical injury $\geq \mathrm{C} 5$ participants) will be included in the study, presumably reducing the number of included individuals with impaired BP regulation. Nevertheless, 
in addition to reduced vasoconstrictor drive, the ability to increase cardiac output is limited in individuals with SCI $\geq \mathrm{T} 5$-T6 with interrupted cardiac sympathetic innervation, which could affect arterial BP stabilisation during conditions in which peripheral vascular resistance is further reduced (ie, during exercise). Therefore, to identify any participants who may be prone to experiencing postexercise hypotension, $\mathrm{BA} \mathrm{BP}$ will be monitored immediately after, and again 5 min after the first $\mathrm{VO}_{\text {2peak }}$ test. In such cases, and assuming the hypotension is well tolerated, the participants will be reminded to drink appropriately during and prior to any future training and testing. Further, these participants will have their BP measured every second interval (ie, every 10th min) during the training sessions. If hypotension develops, the training will be interrupted and hypotensive counter manoeuvres (placement in a supine position, or legs up) will be applied. If the hypotension is not tolerated, such as if the participants exhibit signs of nausea, light-headedness, fatigue or presyncobal symptoms in response to either the $\mathrm{VO}_{\text {2peak }}$ test or the training, they will be excluded from further participation in the study.

Another consideration is that individuals with SCI suffer from impaired thermoregulation. ${ }^{106}$ Although prolonged $(60 \mathrm{~min})$ moderate-intensity exercise in warm conditions $\left(>31^{\circ} \mathrm{C}\right)$ have been shown sustainable for some paraplegic and tetraplegic individuals, ${ }^{107}$ impaired thermoregulation makes individuals with SCI more vulnerable to overheating compared with ablebodied. ${ }^{106}$ This is especially the case during conditions of increased environmental temperatures and metabolic heat production (such as when exercising in the heat). The degree of thermoregulatory impairment is closely related to the injury level, such that individuals with higher injury (tetraplegics) exhibit higher core temperatures during exercise compared with lower injury individuals (paraplegics). ${ }^{107}$ To diminish the risk for overheating during testing and training, a fan will be available for cooling of the participants and the room temperature and humidity will be continuously monitored to facilitate the conditions for dry and evaporative heat loss. Moreover, the participants will be asked to pay attention to proper hydration before and throughout each training session. However, if the participants demonstrate signs of any adverse response, the exercise session will be terminated immediately and further participation in the study will be reconsidered. Any adverse responses from the intervention will be reported to the regional health research committee. The trial is reported to the Danish Data Protection Agency ( J.nr. 2019-899/10-0406), registered at ClinicalTrials.gov on 15 May 2020, and approved by the Committees on Health Research Ethics in The North Denmark Region on 12 December 2019 (Journal-nr. N-20190053). Results will be submitted to scientific journals related to exercise and SCI for publication irrespective of study outcomes.

\section{Data statement section}

All participant data will be stored in a secure web-based database (Redcap) with restricted access and ID code, in accordance with data protection rules. Source documents including date, visit\# and participant ID will be scanned and saved as electronic copies. Participant data will be transferred directly or by use of an encrypted Universal Serial Bus (USB) stick. Each participant will be assigned an unique identification number, which will be the only identifier exported from Redcap on data analysis. Except for the blood samples, which will be stored until analysis, or no more than 5 years, data will be stored for 5 years after the termination of the trial. After this period, the paper material is shredded, data files are erased and the Redcap database is no longer accessible. The principal investigator will have access to all trial data.

\section{Author affiliations}

${ }^{1}$ Sport Sciences - Performance and Technology, Department of Health Science and Technology, Aalborg University, Aalborg, Denmark

${ }^{2}$ Department of Research and Development, University College of Northern Jutland (UCN), Aalborg, Denmark

${ }^{3}$ Physical Therapy Department, University College of Northern Jutland (UCN), Aalborg, Denmark

${ }^{4}$ Department of Clinical Biochemistry, Aalborg University Hospital, Aalborg, Denmark ${ }^{5}$ Department of Clinical Medicine, Aalborg University, Aalborg, Denmark

\section{Twitter Rasmus Kopp Hansen @RKHansen1990}

Acknowledgements The authors would like to thank Dr. Rachel E. Cowan for valuable discussions regarding the study protocol, Drs. Stefanos Volianitis and Pascal Madeleine for their contribution in the initial phase of the project, Dr. Jan Christian Brønd for discussions regarding assessment of leisure time physical activity level, and Wolturnus A/S for lending of arm ergometer and wheelchair weight.

Contributors RKH and RGL conceptualised the study. RKH, AS, UL, AH and RGL contributed to the study protocol design. RKH drafted the manuscript. RKH, AS, UL, $\mathrm{AH}$ and RGL commented and edited the manuscript and approved the final version.

Funding This work is supported by Aage and Johanne Louis-Hansens Fond, grant number 20-2B-5947.

Disclaimer Aage and Johanne Louis-Hansens Fond will have no influence on any part of the project design, data collection, data analysis, data interpretation or the disclosure of the results. Thus, their support is solely economical.

\section{Competing interests None declared.}

Patient consent for publication Not required.

Provenance and peer review Not commissioned; externally peer reviewed.

Supplemental material This content has been supplied by the author(s). It has not been vetted by BMJ Publishing Group Limited (BMJ) and may not have been peer-reviewed. Any opinions or recommendations discussed are solely those of the author(s) and are not endorsed by BMJ. BMJ disclaims all liability and responsibility arising from any reliance placed on the content. Where the content includes any translated material, BMJ does not warrant the accuracy and reliability of the translations (including but not limited to local regulations, clinical guidelines, terminology, drug names and drug dosages), and is not responsible for any error and/or omissions arising from translation and adaptation or otherwise.

Open access This is an open access article distributed in accordance with the Creative Commons Attribution Non Commercial (CC BY-NC 4.0) license, which permits others to distribute, remix, adapt, build upon this work non-commercially, and license their derivative works on different terms, provided the original work is properly cited, appropriate credit is given, any changes made indicated, and the use is non-commercial. See: http://creativecommons.org/licenses/by-nc/4.0/.

ORCID iD

Rasmus Kopp Hansen http://orcid.org/0000-0001-8515-8779 


\section{REFERENCES}

1 LaPorte RE, Adams LL, Savage DD, et al. The spectrum of physical activity, cardiovascular disease and health: an epidemiologic perspective. Am J Epidemiol 1984;120:507-17.

2 Myers J, Lee M, Kiratli J. Cardiovascular disease in spinal cord injury: an overview of prevalence, risk, evaluation, and management. Am J Phys Med Rehabil 2007;86:142-52.

3 Cowan RE, Nash MS. Cardiovascular disease, SCI and exercise: unique risks and focused countermeasures. Disabil Rehabil 2010;32:2228-36.

4 Garshick E, Kelley A, Cohen SA, et al. A prospective assessment of mortality in chronic spinal cord injury. Spinal Cord 2005;43:408-16.

5 Buchholz AC, Martin Ginis KA, Bray SR, et al. Greater daily leisure time physical activity is associated with lower chronic disease risk in adults with spinal cord injury. Appl Physiol Nutr Metab 2009;34:640-7.

6 Gorgey AS, Dolbow DR, Dolbow JD, et al. Effects of spinal cord injury on body composition and metabolic profile - part I. J Spinal Cord Med 2014;37:693-702.

7 Ahuja CS, Wilson JR, Nori S, et al. Traumatic spinal cord injury. Nat Rev Dis Primers 2017;3:1-21.

8 Kirshblum SC, Burns SP, Biering-Sorensen F, et al. International standards for neurological classification of spinal cord injury (revised 2011). J Spinal Cord Med 2011;34:535-46.

9 Haisma JA, van der Woude LHV, Stam HJ, et al. Physical capacity in wheelchair-dependent persons with a spinal cord injury: a critical review of the literature. Spinal Cord 2006;44:642-52.

10 Groah SL, Weitzenkamp D, Sett P, et al. The relationship between neurological level of injury and symptomatic cardiovascular disease risk in the aging spinal injured. Spinal Cord 2001;39:310-7.

11 Manual Wheelchairs Practitioner. Spinal cord injury research evidence (SCIRE) community, 2020. Available: www.scireproject. com/community/topic/manual-wheelchairs/\%0AManual

12 Kehn M, Kroll T. Staying physically active after spinal cord injury: a qualitative exploration of barriers and facilitators to exercise participation. BMC Public Health 2009;9:1-11.

13 Rimmer JH, Riley B, Wang E, et al. Physical activity participation among persons with disabilities: barriers and facilitators. Am J Prev Med 2004;26:419-25.

14 Hansen RK, Larsen RG, Laessoe U, et al. Physical Activity Barriers in Danish Manual Wheelchair Users - A Cross-sectional Study. Archives of Physical Medicine and Rehabilitation, accepted manuscript 21.09. 2020.

15 Cowan RE, Nash MS, Anderson KD. Exercise participation barrier prevalence and association with exercise participation status in individuals with spinal cord injury. Spinal Cord 2013;51:27-32.

16 Rimmer JH, Rubin SS, Braddock D. Barriers to exercise in African American women with physical disabilities. Arch Phys Med Rehabil 2000;81:182-8.

17 Rimmer JH, Wang E, Smith D. Barriers associated with exercise and community access for individuals with stroke. J Rehabil Res Dev 2008;45:315-22.

18 van der Scheer JW, Martin Ginis KA, Ditor DS, et al. Effects of exercise on fitness and health of adults with spinal cord injury: a systematic review. Neurology 2017;89:736-45.

19 El-Sayed MS, Younesian A. Lipid profiles are influenced by arm cranking exercise and training in individuals with spinal cord injury. Spinal Cord 2005;43:299-305.

20 Nightingale TE, Walhin J-P, Thompson D, et al. Impact of exercise on cardiometabolic component risks in spinal cord-injured humans. Med Sci Sports Exerc 2017;49:2469-77.

21 Kim D-I, Lee H, Lee B-S, et al. Effects of a 6-week indoor HandBike exercise program on health and fitness levels in people with spinal cord injury: a randomized controlled trial study. Arch Phys Med Rehabil 2015;96:2033-40.

22 Totosy de Zepetnek JO, Pelletier CA, Hicks AL, et al. Following the physical activity guidelines for adults with spinal cord injury for 16 weeks does not improve vascular health: a randomized controlled trial. Arch Phys Med Rehabil 2015;96:1566-75.

23 Wilt TJ, Carlson KF, Goldish GD, et al. Carbohydrate and lipid disorders and relevant considerations in persons with spinal cord injury. Evid Rep Technol Assess 2008;171:1-95.

24 Pelletier CA, Omidvar M, Miyatani M, et al. Participation in moderate-to-vigorous leisure time physical activity is related to decreased visceral adipose tissue in adults with spinal cord injury. Appl Physiol Nutr Metab 2018;43:139-44.

25 Nightingale TE, Metcalfe RS, Vollaard NB, et al. Exercise guidelines to promote cardiometabolic health in spinal cord injured humans: time to raise the intensity? Arch Phys Med Rehabil 2017;98:1693-704.
26 Hicks AL, Martin Ginis KA, Pelletier CA, et al. The effects of exercise training on physical capacity, strength, body composition and functional performance among adults with spinal cord injury: a systematic review. Spinal Cord 2011:49:1103-27.

27 Green DJ, Driscoll GO, Joyner MJ. Exercise and cardiovascular risk reduction : time to update the rationale for exercise ? 2013: 766-8.

28 Gielen S, Schuler G, Hambrecht R. Exercise training in coronary artery disease and coronary vasomotion. Circulation 2001;103:1-6.

29 Celermajer DS, Sorensen KE, Gooch VM, et al. Non-invasive detection of endothelial dysfunction in children and adults at risk of atherosclerosis. Lancet 1992;340:1111-5.

30 Yeboah J, Folsom AR, Burke GL, et al. Predictive value of brachial flow-mediated dilation for incident cardiovascular events in a population-based study: the multi-ethnic study of atherosclerosis. Circulation 2009;120:502-9.

31 Lorenz MW, Markus HS, Bots ML, et al. Prediction of clinical cardiovascular events with carotid intima-media thickness: a systematic review and meta-analysis. Circulation 2007;115:459-67.

32 Green DJ, Hopman MTE, Padilla J, et al. Vascular adaptation to exercise in humans: role of hemodynamic stimuli. Physiol Rev 2017;97:495-528.

33 de Groot PC, Bleeker MW, van Kuppevelt DH, et al. Rapid and extensive arterial adaptations after spinal cord injury. Arch Phys Med Rehabil 2006;87:688-96.

34 de Groot PCE, Poelkens F, Kooijman M, et al. Preserved flowmediated dilation in the inactive legs of spinal cord-injured individuals. Am J Physiol Heart Circ Physiol 2004;287:H374-80.

35 Huonker M, Schmid A, Schmidt-Trucksass A, et al. Size and blood flow of central and peripheral arteries in highly trained able-bodied and disabled athletes. J Appl Physiol 2003;95:685-91.

36 Rowley NJ, Dawson EA, Hopman MTE, et al. Conduit diameter and wall remodeling in elite athletes and spinal cord injury. Med Sci Sports Exerc 2012;44:844-9.

37 Ginis KAM, Hicks AL, Latimer AE, et al. The development of evidence-informed physical activity guidelines for adults with spinal cord injury. Spinal Cord 2011;49:1088-96.

38 Pelletier CA, Totosy de Zepetnek JO, MacDonald MJ, et al. A 16-week randomized controlled trial evaluating the physical activity guidelines for adults with spinal cord injury. Spinal Cord 2015;53:363-7.

39 Martin Ginis KA, van der Scheer JW, Latimer-Cheung AE, et al. Evidence-based scientific exercise guidelines for adults with spinal cord injury: an update and a new guideline. Spinal Cord 2018;56:308-21.

40 Cratsenberg KA, Deitrick CE, Harrington TK, et al. Effectiveness of exercise programs for management of shoulder pain in manual wheelchair users with spinal cord injury. J Neurol Phys Ther 2015;39:197-203

41 Troy KL, Munce TA, Longworth JA. An exercise trial targeting posterior shoulder strength in manual wheelchair users: pilot results and lessons learned. Disabil Rehabil Assist Technol 2015;10:415-20.

42 Nash MS, Jacobs PL, Woods JM, et al. A comparison of 2 circuit exercise training techniques for eliciting matched metabolic responses in persons with paraplegia. Arch Phys Med Rehabil 2002;83:201-9.

43 Curtis KA, Tyner TM, Zachary L, et al. Effect of a standard exercise protocol on shoulder pain in long-term wheelchair users. Spinal Cord 1999;37:421-9.

44 Ballinger DA, Rintala DH, Hart KA. The relation of shoulder pain and range-of-motion problems to functional limitations, disability, and perceived health of men with spinal cord injury: a multifaceted longitudinal study. Arch Phys Med Rehabil 2000;81:1575-81.

45 Nichols PJ, Norman PA, Ennis JR. Wheelchair user's shoulder? shoulder pain in patients with spinal cord lesions. Scand J Rehabil Med 1979;11:29-32.

46 Gutierrez DD, Thompson L, Kemp B, et al. The relationship of shoulder pain intensity to quality of life, physical activity, and community participation in persons with paraplegia. J Spinal Cord Med 2007;30:251-5.

47 Nash MS, van de Ven I, van Elk N, et al. Effects of circuit resistance training on fitness attributes and upper-extremity pain in middleaged men with paraplegia. Arch Phys Med Rehabil 2007;88:70-5.

48 Leduc BE, Lepage Y. Health-related quality of life after spinal cord injury. Disabil Rehabil 2002;24:196-202.

49 Lidal IB, Veenstra M, Hjeltnes N, et al. Health-related quality of life in persons with long-standing spinal cord injury. Spinal Cord 2008;46:710-5.

50 Ambrosio F, Boninger ML, Souza AL, et al. Biomechanics and strength of manual wheelchair users. J Spinal Cord Med 2005;28:407-14. 
51 Curtis KA, Roach KE, Applegate EB, et al. Reliability and validity of the wheelchair user's shoulder pain index (WUSPI). Paraplegia 1995;33:595-601.

52 Burnham RS, May L, Nelson E, et al. Shoulder pain in wheelchair athletes. The role of muscle imbalance. Am J Sports Med 1993;21:238-42.

53 Bayley JC, Cochran TP, Sledge CB. The weight-bearing shoulder The impingement syndrome in paraplegics. J Bone Joint Surg Am 1987;69:676-8.

54 Collinger JL, Boninger ML, Koontz AM, et al. Shoulder biomechanics during the push phase of wheelchair propulsion: a multisite study of persons with paraplegia. Arch Phys Med Rehabil 2008;89:667-76.

55 Mulroy SJ, Thompson L, Kemp B, et al. Strengthening and optimal movements for painful shoulders (STOMPS) in chronic spinal cord injury: a randomized controlled trial. Phys Ther 2011;91:305-24.

56 Secher NH. The physiology of rowing. J Sports Sci 1983;1:23-53.

57 Olenik LM, Laskin JJ, Burnham R, et al. Efficacy of rowing, backward wheeling and isolated scapular retractor exercise as remedial strength activities for wheelchair users: application of electromyography. Paraplegia 1995;33:148-52.

58 Chan A-W, Tetzlaff JM, Altman DG, et al. Spirit 2013 statement: defining standard protocol items for clinical trials. Ann Intern Med 2013;158:200

59 Lee RE, McGinnis KA, Sallis JF, et al. Active vs. passive methods of recruiting ethnic minority women to a health promotion program. Ann Behav Med 1997;19:378-84.

60 Ginis KAM, Hicks AL. Exercise research issues in the spinal cord injured population. Exerc Sport Sci Rev 2005;33:49-53.

61 Astorino TA, Thum JS, Thum. Interval training elicits higher enjoyment versus moderate exercise in persons with spinal cord injury. J Spinal Cord Med 2018;41:77-84

62 Powell KE, Paluch AE, Blair SN. Physical activity for health: what kind? how much? how intense? on top of what? Annu Rev Public Health 2011;32:349-65.

63 Borg G. Perceived exertion as an indicator of somatic stress. Scand $J$ Rehabil Med 1970;2:92-8.

64 Birk TJ, Birk CA. Use of ratings of perceived exertion for exercise prescription. Sports Med 1987;4:1-8.

65 Goosey-Tolfrey V, Lenton J, Goddard J, et al. Regulating intensity using perceived exertion in spinal cord-injured participants. Med Sci Sports Exerc 2010;42:608-13.

66 van der Scheer JW, Hutchinson MJ, Paulson T, et al. Reliability and validity of subjective measures of aerobic intensity in adults with spinal cord injury: a systematic review. $P m R$ 2018;10:194-207.

67 Lewis JE, Nash MS, Hamm LF, et al. The relationship between perceived exertion and physiologic indicators of stress during graded arm exercise in persons with spinal cord injuries. Arch Phys Med Rehabil 2007;88:1205-11.

68 Noble BJ, Borg GA, Jacobs I, et al. A category-ratio perceived exertion scale: relationship to blood and muscle lactates and heart rate. Med Sci Sports Exerc 1983;15:523-8.

69 Krassioukov AV, Currie KD, Hubli M, et al. Effects of exercise interventions on cardiovascular health in individuals with chronic, motor complete spinal cord injury: protocol for a randomised controlled trial [Cardiovascular Health/Outcomes: Improvements Created by Exercise and education in SCI (CHOICES) Study]. BMJ Open 2019;9:e023540.

70 Thijssen DHJ, Bruno RM, van Mil ACCM, et al. Expert consensus and evidence-based recommendations for the assessment of flowmediated dilation in humans. Eur Heart J 2019;40:2534-47.

71 Limberg JK, Casey DP, Trinity JD, et al. Assessment of resistance vessel function in human skeletal muscle: guidelines for experimental design, Doppler ultrasound, and pharmacology. Am J Physiol Heart Circ Physiol 2020;318:H301-25

72 Grundy SM, Cleeman JI, Daniels SR, et al. Diagnosis and management of the metabolic syndrome: an American heart Association/National heart, lung, and blood Institute scientific statement. Circulation 2005;112:2735-52.

73 Nash MS, Groah SL, Gater DR, et al. Identification and management of cardiometabolic risk after spinal cord injury: clinical practice guideline for health care providers. Top Spinal Cord Inj Rehabil 2018;24:379-423.

74 McCauley LS, Sumrell RM, Gorgey AS. Anthropometric prediction of visceral adipose tissue in persons with motor complete spinal cord injury. Pm R 2018;10:817-25.

75 Edwards LA, Bugaresti JM, Buchholz AC. The amount of visceral adipose tissue and the ratio of visceral to subcutaneous adipose tissue is greater in adults with vs. without spinal cord injury. Faseb $J$ 2007;21:A7-8.
76 Sumrell RM, Nightingale TE, McCauley LS, et al. Anthropometric cutoffs and associations with visceral adiposity and metabolic biomarkers after spinal cord injury. PLoS One 2018;13:e0203049.

77 Joyner MJ, Green DJ. Exercise protects the cardiovascular system: effects beyond traditional risk factors. J Physiol 2009;587:5551-8.

78 West CR, Romer LM, Krassioukov A. Autonomic function and exercise performance in elite athletes with cervical spinal cord injury. Med Sci Sports Exerc 2013;45:261-7.

79 Otsuka Y, Shima N, Moritani T, et al. Orthostatic influence on heart rate and blood pressure variability in trained persons with tetraplegia. Eur J Appl Physiol 2008;104:75-8.

80 Malik M, Bigger JT, Camm AJ, et al. Heart rate variability: standards of measurement, physiological interpretation, and clinical use. Eur Heart J 1996;17:354-81.

81 Shaffer F, Ginsberg JP. An overview of heart rate variability metrics and norms. Front Public Health 2017;5:1-17.

82 Pelletier CA, Jones G, Latimer-Cheung AE, et al. Aerobic capacity, orthostatic tolerance, and exercise perceptions at discharge from inpatient spinal cord injury rehabilitation. Arch Phys Med Rehabil 2013;94:2013-9.

83 Consensus statement on the definition of orthostatic hypotension pure autonomic failure, and multiple system atrophy. The consensus Committee of the American autonomic Society and the American Academy of Neurology. Neurology 1996;46:1470.

84 Thijssen DHJ, Black MA, Pyke KE, et al. Assessment of flowmediated dilation in humans: a methodological and physiological guideline. Am J Physiol Heart Circ Physiol 2011;300:H2-12.

85 Pyke KE, Tschakovsky ME. Peak vs. total reactive hyperemia: which determines the magnitude of flow-mediated dilation? J Appl Physiol 2007;102:1510-9.

86 Padilla J, Johnson BD, Newcomer SC, et al. Normalization of flowmediated dilation to shear stress area under the curve eliminates the impact of variable hyperemic stimulus. Cardiovasc Ultrasound 2008;6:44.

87 Thrush A, Hartshorne T. Vascular ultrasound - how, when and why. Churchill Livingstone, 2010.

88 Maher JL, Cowan RE. Comparison of 1- versus 3-Minute stage duration during arm Ergometry in individuals with spinal cord injury. Arch Phys Med Rehabil 2016;97:1895-900.

89 Eerden S, Dekker R, Hettinga FJ. Maximal and submaximal aerobic tests for wheelchair-dependent persons with spinal cord injury: a systematic review to summarize and identify useful applications for clinical rehabilitation. Disabil Rehabil 2018;40:497-521.

90 Au JS, Totosy DE Zepetnek JO, Macdonald MJ. Modeling perceived exertion during graded arm cycling exercise in spinal cord injury. Med Sci Sports Exerc 2017;49:1190-6.

91 Kouwijzer I, Cowan RE, Maher JL, et al. Interrater and intrarater reliability of ventilatory thresholds determined in individuals with spinal cord injury. Spinal Cord 2019;57:669-78.

92 Leicht CA, Griggs KE, Lavin J, et al. Blood lactate and ventilatory thresholds in wheelchair athletes with tetraplegia and paraplegia. Eur J Appl Physiol 2014;114:1635-43.

93 Larsen C, Hansen S, Hansen L. Translation and cross-cultural adaptation of the Danish version: wheelchair users shoulder pain index (WUSPI), 2015

94 Curtis KA, Roach KE, Applegate EB, et al. Development of the wheelchair user's shoulder pain index (WUSPI). Paraplegia 1995;33:290-3.

95 Curtis KA, Drysdale GA, Lanza RD, et al. Shoulder pain in wheelchair users with tetraplegia and paraplegia. Arch Phys Med Rehabil 1999;80:453-7.

96 Bjorner JB, Thunedborg K, Kristensen TS, et al. The Danish SF-36 health survey: translation and preliminary validity studies. J Clin Epidemiol 1998;51:991-9.

97 Ware JE, Sherbourne CD. The mos 36-item short-form health survey (SF-36). I. conceptual framework and item selection. Med Care 1992;30:473-83.

98 Tate DG, Kalpakjian CZ, Forchheimer MB. Quality of life issues in individuals with spinal cord injury. Arch Phys Med Rehabil 2002;83:S18-25

99 RAND HC. 36-Item short form survey (SF-36) scoring instructions. Available: https://www.rand.org/health-care/surveys tools/mos/36item-short-form/scoring.html

100 Nightingale TE, Rouse PC, Walhin J-P, et al. Home-based exercise enhances health-related quality of life in persons with spinal cord injury: A randomized controlled trial. Arch Phys Med Rehabil 2018:99:1998-2006.

101 Martin Ginis KA, Phang SH, Latimer AE, et al. Reliability and validity tests of the leisure time physical activity questionnaire for people with spinal cord injury. Arch Phys Med Rehabil 2012:93:677-82. 
102 Visovsky C, Corripio J. Feasibility and acceptability of a resistance exercise intervention: for women undergoing chemotherapy for breast cancer. Nurs Health 2015;3:110-9.

103 Claydon VE, Krassioukov AV. Orthostatic hypotension and autonomic pathways after spinal cord injury. J Neurotrauma 2006;23:1713-25.

104 Claydon VE, Hol AT, Eng JJ, et al. Cardiovascular responses and postexercise hypotension after arm cycling exercise in subjects with spinal cord injury. Arch Phys Med Rehabil 2006;87:1106-14.
105 Phillips AA, Krassioukov AV. Contemporary cardiovascular concerns after spinal cord injury: mechanisms, Maladaptations, and management. J Neurotrauma 2015;32:1927-42.

106 Sawka MN, Latzka WA, Pandolf KB. Temperature regulation during upper body exercise: able-bodied and spinal cord injured. Med Sci Sports Exerc 1989;21:132-40.

107 Price MJ, Campbell IG. Effects of spinal cord lesion level upon thermoregulation during exercise in the heat. Med Sci Sports Exerc 2003;35:1100-7. 